\title{
Rotation effects on the convection of binary alloys unidirectionally solidified from below
}

\author{
JAY W. LU and FALIN CHEN $\dagger$ \\ Institute of Applied Mechanics, National Taiwan University, Taipei, Taiwan 10764, \\ Republic of China
}

(Received 9 November 1995 and in final form 28 March 1996)

\begin{abstract}
Rotation used to be thought as an effective method to prohibit the formation of freckles of a binary alloy unidirectionally solidified from below, which we will show in the present paper is true only under restrictive conditions. A linear stability analysis is implemented for the system consisting a dendritic mushy region underlying a bulk fluid region and rotating uniformly about a vertical axis. Emphasis is placed on the rotation effects on the competition between the convection modes, which has a direct influence on the formation of freckles. Results show that rotation generally enhances the stability of the convection modes, but the enhancement is not significant until the rotation speed is very high (such as $10^{5} \mathrm{rpm}$ ), which in some sense agrees well with the previous numerical and experimental results. In spite of its ineffectiveness as an inhibitory method to the formation of freckles, it is found from the present parametric study that the effects of Iutation on convection depend largely on the buoyancy ratio $\mathscr{A}$ of the alloy. Both the changes of the rotation speed and the buoyancy ratio may lead to a switch of the most critical convection mode, which may in turn influence the characteristics of the subsequent plume convection, and thus the formation of freckles. Copyright ic: 1996 Elsevier Science l td.
\end{abstract}

\section{INTRODUCTION}

The chimney-like plume convection in directional solidification of binary alloys has attracted great research efforts in the past three decades (see, for example, refs. [1-13] and the references therein) owing to its analogy to the freckle (or macrosegregation), which has a detrimental influence on the mechanical strength of castings. The formation of plumes (or freckles), as pointed out in a weakly nonlinear analysis by Amberg and Homsy [10], can be a result of the mushy-layer mode convection, a convection circulating between the bulk fluid and the mushy regions; which was also supported by the experiment of Tait and Jaupart [7]. In a study including both theoretical and experimental approaches, Chen $e t$ al. [13] found that the salt-finger type boundary-layer mode convection, a convection of small wavelength prevailing above the melt-mush interface, is generally more unstable than the mushylayer mode convection, as used to be observed in experiments. They, nevertheless, inferred further that the plumes obscrved in experiment are induced by the subcritical mushy-layer mode convection, which occurs under the strong perturbation imposed by the nonlinear salt-finger convection. As a result, the upward flow of the mushy-layer mode convection carrying colder and less concentrated fluid equilibrates its temperature with the surrounding fluid in the mush, leading to the local remelting of crystal and the formation of plumes.

† Author to whom correspondence should be addressed.
Although in the experiment the boundary-layer mode convection used to occur prior to the mushylayer mode convection, under some circumstances, however, the latter can be more unstable than the former [13]. For example, for an alloy of small $\varepsilon$ (the inverse of Lewis number) or for an alloy having a dendritic mush of large permeability, the onset of convection is largely confined to the mushy region and therefore the mushy-layer mode prevails. Consequently, the plume convection may occur without saltfinger beforehand. In such a case, the onset of plume convection is linear, which occurs under small perturbation. The competition between the boundarylayer and mushy-layer modes decides the sequence of the occurrence of the two convection modes, directly influencing the characteristics of the subsequent plume convection and thus the formation of freckles.

Because of the deleterious effects of freckles on the strength of alloys, the mcasures to prevent the formation of freckles became a major goal to reach in solidification research. In terms of the inhibitory method, rotation has been one of the choices for quite a long time. The effects of rotation on solidification was first discussed by Shulz-DuBois [14]. Kuo et al. [15] conducted experiments on $\mathrm{Pb}-\mathrm{Sn}$ alloys and found that modest rotation speed about a vertical axis can reduce macrosegregation in all ingots studied. Sample and Hellawell [3] rotated the casting mode about an axis inclined between 0 and $30^{\circ}$, effectively prevented the formation of freckles under a very low rotating speed (less than $5 \mathrm{rpm}$ ). In an analytical study, Oztekin and Pearlstein [16] discussed the Cori- 


\section{NOMENCLATURE}

C concentration

$C_{\mathrm{E}} \quad$ concentration of eutectic solid

$C_{\infty} \quad$ concentration at $z=\infty$

$C_{\mathrm{S}} \quad$ concentration of solid

$C_{\mathrm{p}} \quad$ specific heat

$D$ differential operator $\mathrm{d} / \mathrm{d} z$

$D_{f} \quad$ solutal diffusivity

$\mathbf{e}_{z} \quad$ unit vector of vertical axis

$g$ gravitational acceleration constant

$H \quad$ characteristic length $\kappa_{\mathrm{f}} / V$

$h \quad$ height of mushy region

$L \quad$ latent heat of melt

n unit vector normal to the interface

$p \quad$ pressure

$R_{\text {c }} \quad$ solutal Rayleigh number of the fluid region, equation (7)

$R_{\mathrm{t}} \quad$ thermal Rayleigh number of the fluid region, equation (7)

$R_{\mathrm{m}} \quad$ solutal Rayleigh number of the mushy region, equation ( 7 )

$R_{\mathrm{m}}^{\mathrm{c}} \quad$ critical solutal Rayleigh number

$T$ temperature

$T_{\mathrm{a}} \quad$ Taylor number in the fluid region $\left(2 I I^{2} \Omega_{0} / v\right)^{2}$

$T_{\mathrm{L}} \quad$ liquidus temperature

$T_{\infty} \quad$ temperature at $z=\infty$

$T_{\mathrm{m}} \quad$ Taylor number in the mushy region $\left(2 \Pi_{0} \Omega_{0} / v\right)^{2}$

$t$ time

u velocity vector $(u, v, w)$

$\mathbf{u}_{2} \quad$ planar velocity vector $(u, v)$

$V \quad$ velocity of interface

$W \quad$ small disturbance vertical velocity

$z \quad$ vertical axis.

Greek symbols

$\mathscr{A}$ buoyancy ratio $\Gamma \alpha^{*} / \beta$

$\alpha \quad$ horizontal wave number of normal mode $\alpha^{i} \quad$ critical wave number

$\alpha^{*} \quad$ thermal expansion coefficient

$\beta \quad \beta^{*}-\Gamma \alpha^{*}$

$\beta^{*} \quad$ solutal expansion coefficient

$\mathscr{C}$ concentration ratio $\left(C_{\mathrm{S}}-C_{x_{0}}\right) / \Delta C$

$\chi \quad$ porosity

$\chi_{\mathrm{m}} \quad$ basic state porosity

$\Delta C \quad C_{\propto}-C_{\mathrm{E}}$

$\Delta T \quad \Gamma \Delta C$ or $T_{\mathrm{L}}\left(C_{x_{1}}\right)-T_{\mathrm{E}}$

$\varepsilon \quad$ inverse Lewis number $D_{\mathrm{l}} / \kappa_{\mathrm{f}}$

$\eta \quad$ small disturbance of interface position

$\mathscr{F} \quad$ Stefan number $L /\left(\rho C_{\mathrm{p}}\right)_{\mathrm{f}} \Delta T$

$\mathscr{H} \quad$ Darcy number $H^{2} / \Pi_{0}$

$\kappa_{\mathrm{r}} \quad$ thermal diffusivity

$\Lambda \quad$ Beavers-Joseph constant

$\Gamma \quad$ slope of liquidus, a constant

$v \quad$ kinemalic viscosity

$\Omega_{0} \quad$ angular velocity

$\omega$ frequency of normal mode,

$$
\omega=\omega_{\mathrm{r}}+i \omega_{\mathrm{i}}
$$

$\Pi$ permeability function

$\Pi_{0} \quad$ reference permeahility

$\rho$ density

$\rho_{0} \quad$ reference density

$\sigma \quad$ Prandtl number $v / \kappa_{\mathrm{f}}$

$\Theta \quad$ dimensionless or small disturbance concentration

$\Theta_{\mathrm{f}} \quad$ basic state concentration in the fluid region

$\theta$ dimensionless or small disturbance temperature

$\theta_{\uparrow} \quad$ basic state temperature in the fluid region

$\theta_{\mathrm{j}} \quad$ basic state temperature at the meltmush interface

$\theta_{\infty} \quad$ dimensionless temperature at $z=\infty$

$\theta_{\mathrm{m}} \quad$ basic state temperature in the mushy region

$\zeta \quad$ small disturbance vorticity $\partial v / \partial x-\partial u / \partial y$. olis effect on the convective stability in a plane-front solidification of dilute $\mathrm{Pb}-\mathrm{Sn}$ binary alloy. Neilson and Incropera [17] concluded from a nonlinear computation that the formation of freckles can be prevented when the casting mold is rotating about a vertical axis with a speed-up and speed-down cycle while a steady rotation up to $120 \mathrm{rpm}$ is not sufficient to alter the convective flow.

Previous results $[15,17]$ suggest that small rotation speed cannot effectively prohibit the formation of freckles. Since the consequence of the competition between the two convection modes influences directly the formation of freckles, and under rotation, the competition may change, we thus conduct the present study in which a linear stability analysis is implemented for the convection in the system consisting a dendritic mushy zone and a semi-infinite fiuid region. The system rotates about a vertical axis with a constant angular velocity. The effects of rotation on the stability of the two convection modes will he examined systematically. Physical mechanisms corresponding to the rotation effect on the convection mode will be discussed. In the following paper, the mathematical formulation and small perturbation equations are presented in Sections 2 and 3, respectively. The numerical results and discussion are shown in Section 4 and several concluding remarks are given in Section 5. 


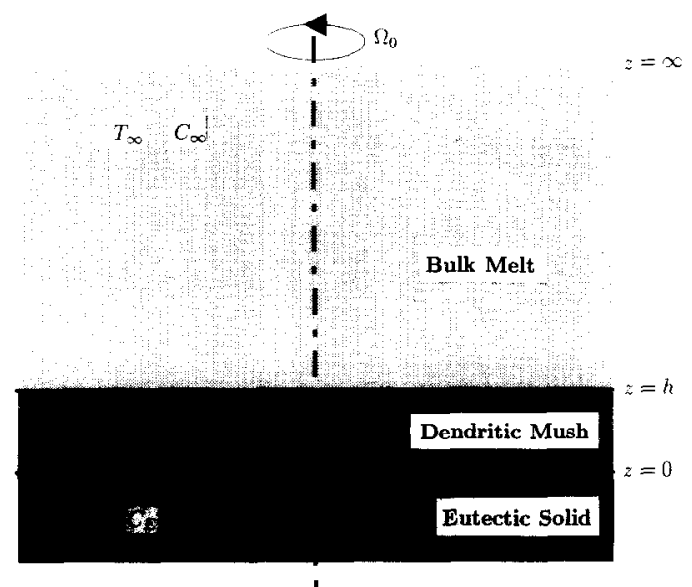

Fig. 1. Schematic description of the physical domain considered.

\section{MATHEMATICAL FORMULATION}

Consider that a binary alloy of concentration $C_{x}\left(>C_{\mathrm{t}}\right)$ and temperature $T_{\infty}$ is unidirectionally solidified from below, in which a dendritic mush forms between the eutectic solid and the semi-infinite bulk fluid (Fig. 1). Assume that both the solid-mush and the mush-fluid interfaces move upwards with a constant velocity $V$ and the system rotates uniformly about the vertical axis $z$ with a constant angular velocity $\Omega_{0}$. To describe the fluid motion in such a system, two sets of equations are required, one for the fluid region and one for the mushy region. Each set includes the conservation of mass, momentum, heat, and solute. In the momentum equations Boussinesq approximation is employed; namely, the density of the fluid is assumed to be constant except in the gravity term, in which the density is a function of temperature and concentration

$$
\rho=\rho_{0}\left\{1-\alpha^{*}\left[T-T_{\mathrm{L}}\left(C_{\alpha}^{\prime}\right)\right]+\beta^{*}\left[C-C_{\infty}\right]\right\} .
$$

We non-dimensionalize the fluid velocities with $V$, distances with $\kappa_{\mathrm{f}} / V$, time with $\kappa_{\mathrm{f}} / V^{2}$, angular velocity with $\Omega_{0}$, and pressure with $\beta \Delta C \rho_{0} g \kappa_{\mathrm{f}} / V$, in which $\beta=\beta^{*}-\Gamma \alpha^{*}$, and $\Delta C=C_{\infty}-C_{\mathrm{E}}$. By taking the Galilean transformation with respect to the moving interface, we obtain the following dimensionless equations. In the fluid region $h<z<\infty$, we have

$$
\begin{gathered}
\nabla \cdot \mathbf{u}=0 \\
\left(\frac{\partial}{\partial t}-\frac{\partial}{\partial z}\right) \theta+\mathbf{u} \cdot \nabla \theta=\nabla^{2} \theta \\
\left(\frac{\partial}{\partial t}-\frac{\partial}{\partial z}\right) \Theta+\mathbf{u} \cdot \nabla \Theta=\varepsilon \nabla^{2} \Theta
\end{gathered}
$$$$
\frac{1}{\sigma}\left[\left(\frac{\partial}{\partial t}-\frac{\partial}{\partial z}\right)+\mathbf{u} \cdot \nabla\right] \mathbf{u}
$$$$
=\nabla^{2} \mathbf{u}+\sqrt{T_{\mathrm{a}}} \mathbf{u} \times \mathbf{e}_{z}+R_{\mathrm{t}} \theta \mathbf{e}_{z}-R_{\mathrm{c}}\left(\Theta \mathbf{e}_{z}+\frac{\beta}{\beta^{*}} \nabla p\right) .
$$

In the above equations, the dimensionless temperature and concentration are defined as

$$
\theta=\frac{T-T_{\mathrm{L}}\left(C_{\infty}\right)}{\Delta T} \quad \Theta=\frac{C-C_{\infty}}{\Delta C}
$$

$\Delta T=\Gamma \Delta C=T_{\mathrm{L}}\left(C_{\infty}\right)-T_{\mathrm{E}}, T_{\mathrm{L}}\left(C_{\infty}\right)$ is the liquidus temperature corresponding to $C_{\infty}, R_{\mathrm{t}}$ and $R_{\mathrm{c}}$ are thermal and solutal Rayleigh numbers given by

$$
R_{\mathrm{t}}=\frac{g \alpha^{*} \Delta T H^{3}}{\kappa_{\mathrm{f}} v} \quad R_{\mathrm{c}}=\frac{g \beta^{*} \Delta C H^{3}}{\kappa_{\mathrm{f}} v}
$$

and $T_{\mathrm{a}}=\left(2 H^{2} \Omega_{0} / v\right)^{2}$ is the Taylor number in the fluid region. In the mushy region $0<z<h$, we have

$$
\begin{gathered}
\nabla \cdot \mathbf{u}=0 \\
\left(\frac{\partial}{\partial t}-\frac{\partial}{\partial z}\right) \theta+\mathbf{u} \cdot \nabla \theta=\nabla^{2} \theta-\mathscr{F}\left(\frac{\partial}{\partial t}-\frac{\partial}{\partial z}\right) \chi \\
\chi\left(\frac{\partial}{\partial t}-\frac{\partial}{\partial z}\right) \Theta+\mathbf{u} \cdot \nabla \Theta=-(\Theta-\mathscr{C})\left(\frac{\partial}{\partial t}-\frac{\partial}{\partial z}\right) \chi \\
\frac{\mathbf{u}}{\Pi(\chi)}=\frac{\sqrt{T_{\mathrm{m}}}}{\chi} \mathbf{u} \times \mathbf{e}_{z}-R_{\mathrm{m}}\left(\nabla p+\theta \mathbf{e}_{z}\right)
\end{gathered}
$$

where $\mathscr{C}=\left(C_{\mathrm{S}}-C_{\alpha x}\right) / \Delta C^{-}$is the concentration ratio and $R_{\mathrm{m}}$ is the Rayleigh number for the mushy layer given by

$$
R_{\mathrm{m}}=\frac{g \beta \Delta C \Pi_{0} H}{\kappa_{\mathrm{f}} \nu}
$$

and $T_{\mathrm{m}}=\left(2 \Omega_{0} \Pi_{0} / v\right)^{2}$ is the Taylor number in the mushy region. The Rayleigh numbers in the fluid can be replaced by the Rayleigh number in the mush with the relations $R_{\mathrm{t}}=\mathscr{A} \mathscr{H} R_{\mathrm{m}}$ and $R_{\mathrm{c}}=(1+\mathscr{A}) \mathscr{H} R_{\mathrm{m}}$, where $\mathscr{A}=\Gamma \alpha^{*} / \beta$ is the buoyancy ratio, accounting for the ratio between the buoyancy due to thermal expansion to that due to solutal expansion. The Taylor numbers of the fluid and mushy regions have the relation $T_{\mathrm{a}}=\mathscr{H}^{2} T_{\mathrm{m}}$. The dimensionless permeability function $\Pi(\chi)$ is an arbitrary function of the porosity $\chi$, which we consider $\Pi(\chi)=\chi^{3}$ [8] for the present study. The thermodynamic equilibrium condition holds in the mushy layer [13], in which the liquidus relation $\theta=\Theta$ is applied. The solutal diffusion of the mush is assumed to be zero because it is indecd very small [8].

The boundary conditions at $z \rightarrow \infty$ are

$$
\theta-\theta_{\propto} \quad \Theta=0 \quad \mathbf{u}=0
$$

at the melt-mush interface $z=h$ are

$$
\begin{aligned}
\theta & =\Theta \quad \mathbf{n} \cdot \nabla \theta=\mathbf{n} \cdot \nabla \Theta \quad[\mathbf{n} \cdot \mathbf{u}]=0 \\
{[\theta] } & =0 \quad[\mathbf{n} \cdot \nabla \theta]=0 \quad \chi=1 \quad[p]=0 \\
\left.\frac{\partial \mathbf{u}_{2}}{\partial z}\right|_{h+} & =\Lambda \sqrt{\frac{\mathscr{H}}{\Pi(1)}}\left(\left.\mathbf{u}_{2}\right|_{h+}-\left.\mathbf{u}_{2}\right|_{h-}\right)
\end{aligned}
$$

where the square brackets denote the jump of the enclosed quantity across the interface, and $\mathbf{u}_{2}$ is the 
plane velocity vector $(u, v)$. For the physical meaning of equation (14) the reader is referred to Chen et al. [13]. The constant $\Lambda$ of equation (14) is determined experimentally to be $0.1 \leqslant \Lambda \leqslant 4$ [18], and we assume $\Lambda=0.1$ for the present study. The interface position $h$ is to be determined as part of the solution. At the mush-solid interface $z=0$ we have

$$
\theta=-1 \quad w=0
$$

where $T=T_{\mathrm{E}}$ is assumed and non-penetration condition is applied.

\section{SMALL DISTURBANCE EQUATIONS}

Since rotation does not alter the basic state, the motionless basic state is therefore the same with that shown in, for example, Worster [8]. The equations in Section 2 are linearized by introducing small disturbances to the basic state quantities, substituting their combination into the original equations and neglecting higher order terms of small disturbance quantities. After introducing the normal modes proportional to $\mathrm{e}^{\omega t+i x x}$ into the linearized equations, the small disturbance equations are obtained as follows. In the fluid region, we have

$$
\begin{gathered}
{\left[D^{2}+\frac{1}{\sigma}(D-\omega)-\alpha^{2}\right] \zeta--\sqrt{T_{\mathrm{a}}} D W} \\
{\left[D^{2}+D-\omega-\alpha^{2}\right] \theta=\theta_{\mathrm{f}}^{\prime} W} \\
{\left[\varepsilon D^{2}+D-\omega-\varepsilon \alpha^{2}\right] \Theta=\Theta_{\mathrm{f}}^{\prime} W} \\
{\left[D^{2}+\frac{1}{\sigma}(D-\omega)-\alpha^{2}\right]\left(D^{2}-\alpha^{2}\right) W} \\
=\sqrt{T_{\mathrm{a}}} D \zeta+\alpha^{2}\left[R_{\mathrm{t}} \theta-R_{\mathrm{c}} \Theta\right] .
\end{gathered}
$$

In these equations, the notations $W, \theta, \Theta$ now represent small disturbance quantities, $\theta_{\mathrm{f}}$ and $\Theta_{\mathrm{f}}$ are basic state temperature and concentration, respectively [8], both $D$ and 'denote $\mathrm{d} / \mathrm{d} z$, and $\zeta=\partial v / \partial x-\partial u / \partial y$ is the small disturbance vorticity. Similarly, in the mushy region we have

$$
\begin{array}{r}
\left(D^{2}+D-\omega-\alpha^{2}\right) \theta+\mathscr{F}(D-\omega) \chi=\theta_{\mathrm{m}}^{\prime} W \\
{\left[\chi_{\mathrm{m}}(D-\omega)+\chi_{\mathrm{m}}^{\prime}\right] \theta+\left[\left(\theta_{\mathrm{m}}-\mathscr{C}\right)(D-\omega)+\theta_{\mathrm{m}}^{\prime}\right] \chi=\theta_{\mathrm{m}}^{\prime} W}
\end{array}
$$

$$
\begin{aligned}
{\left[\frac{1}{\Pi(\chi)}\right.} & \left.+T_{\mathrm{m}} \frac{\Pi(\chi)}{\chi^{2}}\right] D^{2} W+\left[\left(\frac{1}{\Pi(\chi)}\right)^{\prime}\right. \\
& \left.+T_{\mathrm{m}}\left(\frac{\Pi(\chi)}{\chi^{2}}\right)^{\prime}\right] D W-\frac{\alpha^{2}}{\Pi(\chi)} W=\alpha^{2} R_{\mathrm{m}} \theta
\end{aligned}
$$

In equation (21) the liquidus relation $\theta=\Theta$ has been applied, $\theta_{\mathrm{m}}, \chi_{\mathrm{m}}$ are basic state solutions in the mushy region [8].

The boundary conditions at $z \rightarrow \infty$ are

$$
\theta-0 \quad \Theta=0 \quad W=0 \quad D W-0 \quad \zeta=0
$$

at $z=h$ are

$$
\begin{gathered}
\theta=\Theta \quad D \Theta-D \theta=\left(\frac{1-\varepsilon}{\varepsilon}\right) \theta_{\mathrm{f}}^{\prime} \eta \quad[W]=0 \\
{[\theta]=0 \quad[D \theta]=-\frac{\mathscr{F} \theta_{f}^{\prime}}{\mathscr{C}-\theta_{\mathrm{i}}} \eta \quad \chi=\frac{\theta_{\mathrm{f}}^{\prime}}{\theta_{\mathrm{i}}-\mathscr{C}} \eta} \\
{\left[\frac{1}{\Pi(1)} D W+\sqrt{T_{\mathrm{m}} \zeta}\right]_{h-}} \\
=-\frac{1}{\mathscr{H}}\left[\left(D^{2}-3 \alpha^{2}\right) D W+\frac{1}{\sigma}(D-\omega) D W-\sqrt{T_{\mathrm{a}}} \zeta\right]_{h+} \\
\left.D^{2} W\right|_{h+}=\Lambda \sqrt{\frac{\mathscr{H}}{\Pi(1)}}\left(\left.D W\right|_{h+}-\left.D W\right|_{h-}\right) \\
\left.D \zeta\right|_{h+}=\Lambda \sqrt{\frac{\mathscr{H}}{I I(1)}}\left(\left.\zeta\right|_{h,}-\left.\sqrt{T_{\mathrm{m}}} D W\right|_{h-}\right)
\end{gathered}
$$

and at $z=0$ are

$$
\theta=0 \quad W=0
$$

where $\eta$ is the small perturbation of the interface position, $\theta_{i}$ is the basic state temperature at the meltmush interface, and $\chi=1$ at the melt-mush interface. Equations (16)-(25) consists of a complex eigenvalue problem, having totally 15 orders of differentiation along with 16 boundary conditions, in which the second part of equation (24) is employed to solve the disturbance melt-mush interface position $\eta$. We solve this complex eigenvalue problem with a shooting method. For details of the numerical method the reader is referred to Chen et al. [13].

\section{RESULTS AND DISCUSSION}

Equations (16)-(25) contains several physical parameters needed to be determined for analysis. In the present paper, we consider $\mathscr{F}=\mathscr{C}=\theta_{\infty}=1, \sigma=10$, $\varepsilon=0.025$ and $\mathscr{H}=10^{5}$ for a special alloy. The physical meaning of the above parameters and their corresponding influence on the stability of convection have been discussed in Chen et al. [13]. The buoyancy ratio $\mathscr{A}$, significantly influencing the stability of the convective modes, is left as a free parameter so that its effect can be studied. The Taylor number, essentially representing the rotation speed, is another parameter to be examined systematically. The Rayleigh number and the wavenumber are the two parameters to reflect the stability of the convection. The frequency $\omega$ of the normal mode accounts for the intrinsic characteristics of the instability mode, which is real when the mode is steady and is complex when the mode is oscillatory.

Physically, the buoyancy ratio $\mathscr{A}$ accounts for the ratio between the buoyancy due to thermal expansion to the buoyancy due to solutal expansion. For $\mathscr{A}=0$, the buoyancy due to thermal expansion disappears from the system; namely, the interaction between 


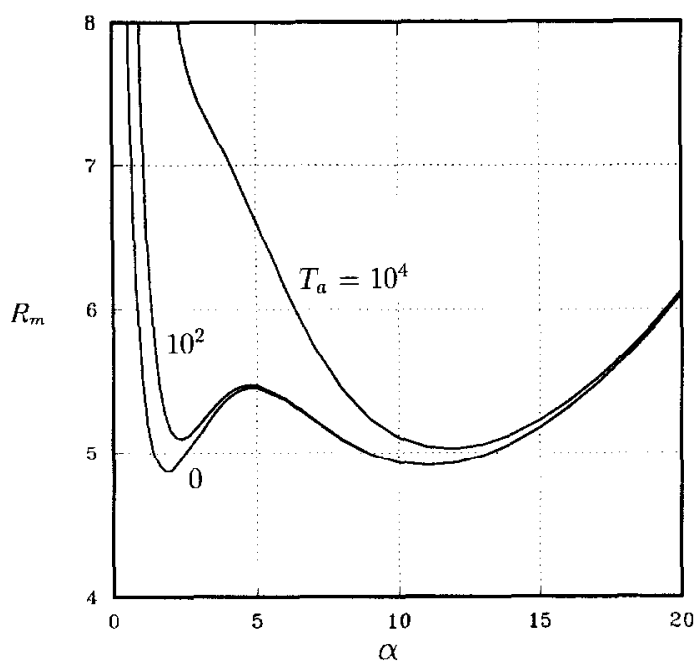

Fig. 2. The neutral curves for various $T_{\mathrm{d}}$ when $\mathscr{A}=0$.

thermal and solutal gradients is eliminated from the system; the flow is a compositional convection. For $\mathscr{A}>0$, both thermal and solutal gradients effectively influence the system and the flow is a double-diffusive convection. For the influence of $\mathscr{A}$ on the stability of the convective modes, Chen et al. [13] concluded that increasing $\mathscr{A}$ enhances the stability of the mushylayer mode which leads to a less stable boundary-layer mode except at very small $\mathscr{A}$. This conclusion in fact becomes more obvious when rotation is applied, which will be discussed in the following.

Figures 2 and $4-6$ illustrate the neutral curves for four different $\mathscr{A}(0,0.1,0.3$ and 0.5$)$ when $T_{\mathrm{a}}$ increases from 0 to about $10^{8}$. For $\mathscr{A}=0$ (Fig. 2), the onset of convection is steady $\left(\omega_{\mathrm{i}}=0\right)$ for all $T_{\mathrm{a}}$ considered. For $T_{\mathrm{a}}=0$, the mushy-layer mode $\left(\alpha^{\mathrm{c}}=1.76\right)$ is more unstable than the boundary-layer mode $\left(\alpha^{\mathrm{c}}=11.1\right)$. Figure 3 shows the onset flow patterns for these two modes. The boundary-layer mode (solid lines) lies

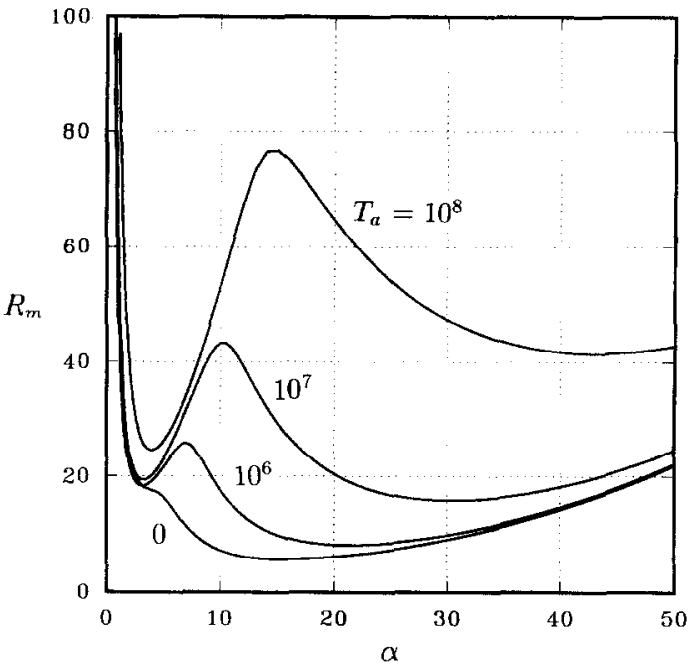

Fig. 4. The neutral curves for various $T_{\mathrm{a}}$ when $\mathscr{A}=0.1$.

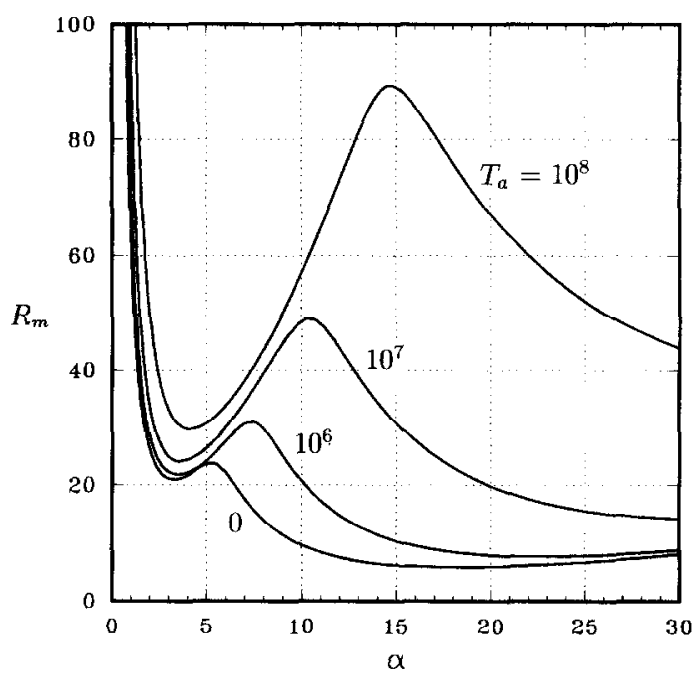

Fig. 5. The neutral curves for various $T_{\mathrm{a}}$ when $\mathscr{A}=0.3$.

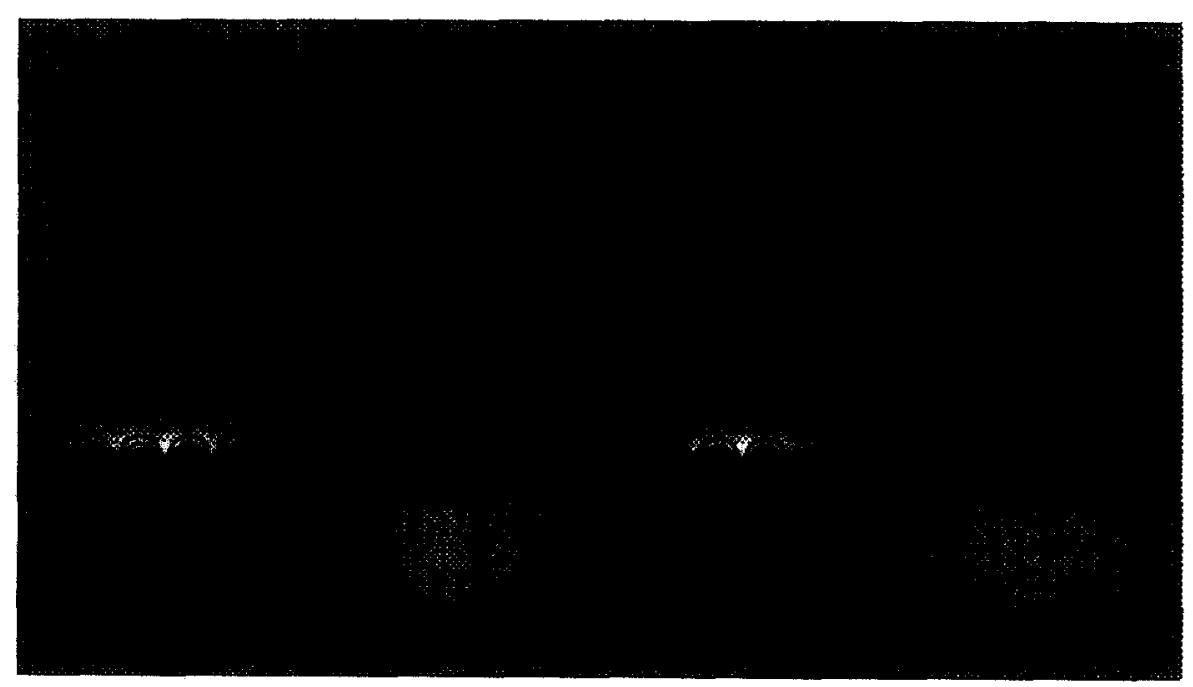

Fig. 3. The onset streamline pattern of the mushy-layer mode convection (shadowed) and the boundarylayer mode convection (solid curve). The mushy-layer lies below the boundary-layer mode cells. The wavelength of the convection mode is scaled with respect to the height of the mushy-layer. 
(a)

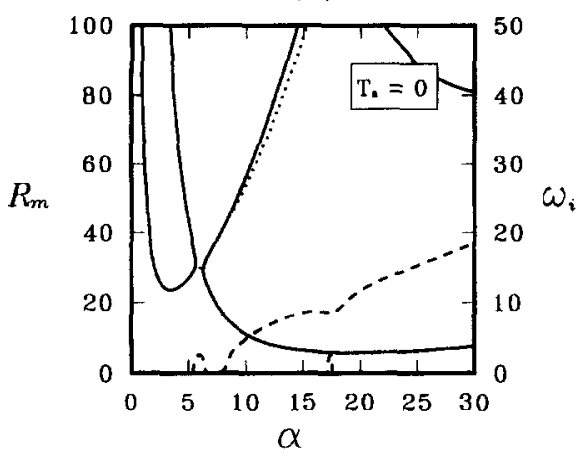

(c)

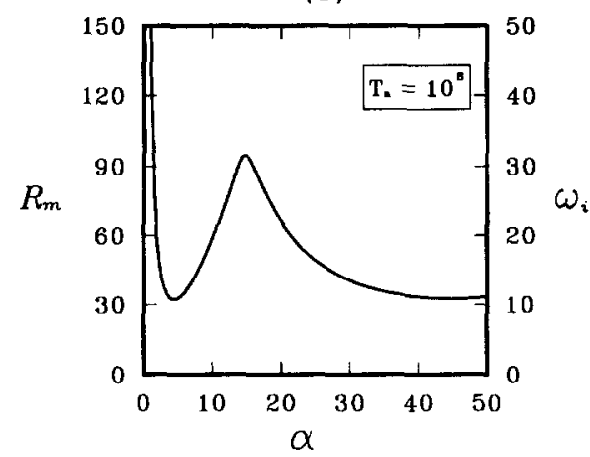

(b)

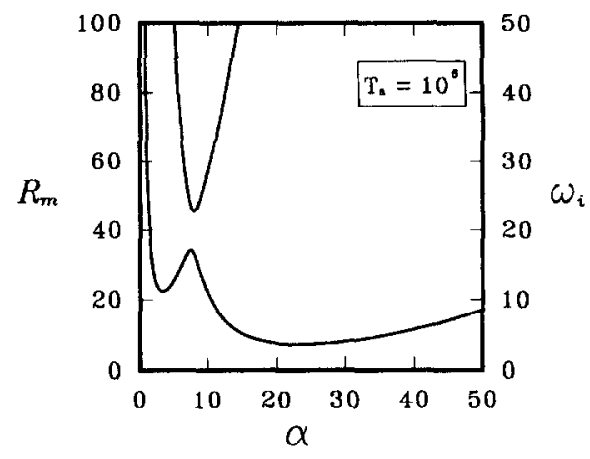

(d)

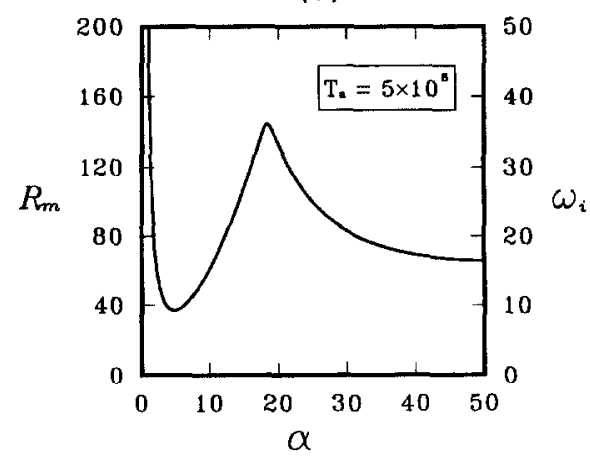

Fig. 6. The neutral curves for various $T_{\mathrm{a}}$ when $\mathscr{A}=0.5$. The solid curves account for the steady mode $\left(\omega_{\mathrm{i}}=0\right)$, the dotted curves account for the oscillatory mode $\left(\omega_{\mathrm{i}} \neq 0\right)$, and the dashed curve accounts for the frequency of the oscillation $\omega_{\mathrm{i}}$.

above the melt-mush interface, having a characteristic length scale about the thickness of the compositional boundary-layer [8]. The mushy-layer mode (shadowed), having a scale about the thickness of the mushy-layer, consists two separated cells, one setting above the melt-mush interface and the other lying mostly within the mush. The two cells circulates in the opposite directions, affecting each other through viscous shear force. As $T_{\mathrm{a}}$ increases to $10^{2}$, the boundary-layer mode becomes more unstable than the mushy-layer mode. For $T_{a}=10^{4}$, the local minimum of the neutral curve for the mushy-layer mode in essence disappears and the boundary-layer mode is the most critical one.

Results of Fig. 2 show that rotation stabilizes both the boundary-layer and the mushy-layer modes while virtually makes no change on their wavelength. This stabilizing effect is more to the latter than to the former. To explain this, we realize that for $\mathscr{A}=0$ the thermal gradient effect is absent from the system and only the solutal gradient is destabilizing the motionless basic state, against the stabilizing viscous force. The destabilizing solutal gradient induces a buoyant flow moving vertically, which is suppressed by rotation through the so-called Taylor-Proudman mechanism, which as described by Chandrasekhar [19] that a steady fluid motion parallel to the axis of rotation is prohibited by rotation. Besides, the vertical motion of the mushy-layer mode convection is reduced more by rotation than that of the boundarylayer mode because the circulation region of the former is larger.

For $\mathscr{A}=0.1$ (Fig. 4), the double-diffusive effect comes into play. Rotation stabilizes more the boundary-layer mode than the mushy-layer mode, reduces largely the wavelength of the boundary-layer mode while makes virtually no change on the mushy-layer mode. Results of Fig. 4 suggest that the stability characteristics for $\mathscr{A}=0.1$ are very different from the case of $\mathscr{A}=0$ while are quite similar to those for $\mathscr{A}=0.3$ (Fig. 5) and $\mathscr{A}=0.5$ (Fig. 6). Special attention should be paid to Fig. 6 when $T_{\mathrm{a}}=0$ the neutral curves of the two modes are separated, but connected by a curve (dotted curve) accounting for the oscillatory mode $\left(\omega_{\mathrm{i}} \neq 0\right)$, which is a Hopf bifurcation of the steady modes. The oscillatory curve penetrates into the region enclosed by the steady curve of the boundary-layer mode, sitting very close to the upper part of the enclosed curve. In the same figure, the dashed curve represents the frequency of the oscillation $\left(\omega_{i}\right)$, which increases with the wave number $\alpha$. As $T_{\mathrm{a}}>0$, the oscillatory curve diminishes, the two steady curves of the mushy-layer mode and the boundary-layer mode join together. The suppression of the 
(a)

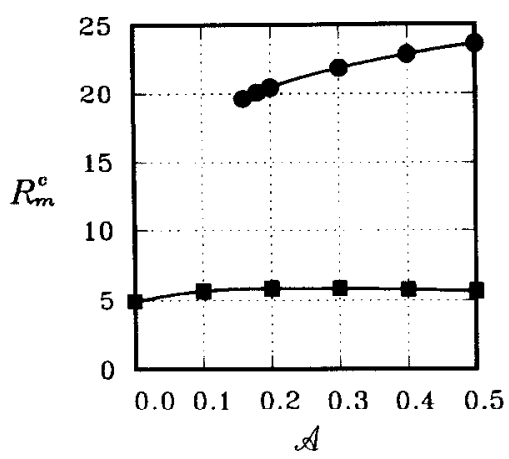

(c)

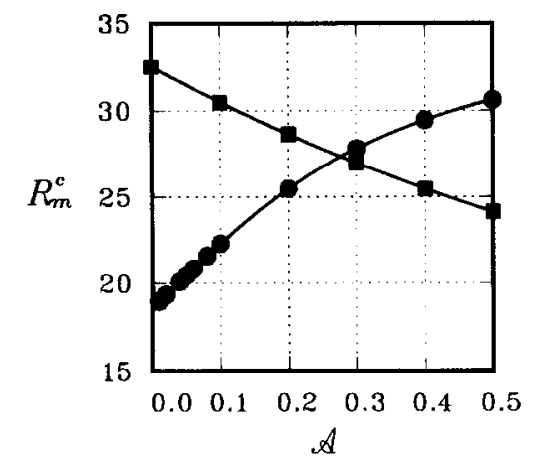

(b)

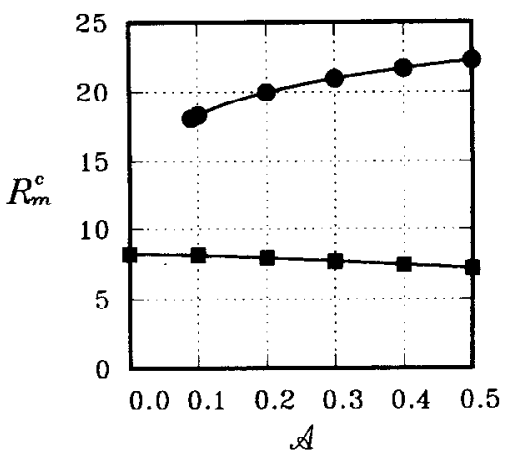

(d)

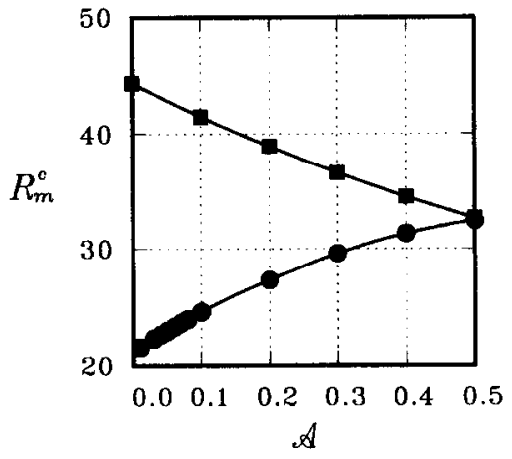

Fig. 7. The variations of $R_{\mathrm{m}}^{\mathrm{c}}$ with $\mathscr{A}$ for four different $T_{\mathrm{a}}:$ (a) $T_{\mathrm{a}}=0 ;$ (b) $T_{\mathrm{a}}=10^{6}$; (c) $T_{\mathrm{a}}=5 \times 10^{7}$; (d) $T_{\mathrm{a}}=10^{8}$. - - the mushy-layer mode; - , the boundary-layer mode.

oscillatory mode convection due to rotation agrees well with the conclusion of Weber et al. [20] that the unsteady convection can be forced to become stcady just by increasing the centrifugal acceleration. Under rotation, both the mushy-layer mode and the boundary-layer mode increase their stability as $T_{\mathrm{a}}$ increases while the latter becomes more stable relative to the former.

To examine the effect of $\mathscr{A}$ under rotation, we show in Fig. 7 the variation of $R_{\mathrm{m}}^{\mathrm{c}}$ (the local minimum of the neutral curve of the convection mode) with $\mathscr{A}$ for four different $T_{\mathrm{a}}\left(0,10^{6}, 5 \times 10^{7}, 10^{8}\right)$. For $T_{\mathrm{a}}=0$ [Fig. 7 (a)], the $R_{\mathrm{m}}^{\mathrm{c}}$ of the mushy-layer mode increases with $\mathscr{A}$ while that of the boundary-layer mode generally decreases slightly with $\mathscr{A}$ except at small $\mathscr{A}$ (see also Chen et al. [13]). To explain this, Worster [8] argued that to the mushy-layer mode large $\mathscr{A}$ means to impose a stable 'lid' on the top of the mushy layer, leading to a more stable state. To the boundary-layer mode, Turner [21] showed that increasing $\mathscr{A}$ tends to stabilize the convection but slightly, since thermal gradient is a stabilizing factor to the system. This, however, is true only for small $\mathscr{A}$ of the present system. For larger $\mathscr{A}$, the boundary-layer mode is destabilized by increasing $\mathscr{A}$ (but also slightly) and this destabilization is even enhanced as rotation is applied. As shown in Figs 7(b)-(d) when rotation is brought into effect, the $R_{\mathrm{m}}^{\mathrm{c}}$ of the mushy-layer mode increases with $\mathscr{A}$ while that of the boundary-layer mode decreases with $\mathscr{A}$; both the increase and the decrease are magnified when $T_{\mathrm{a}}$ is larger. Note that for $T_{\mathrm{a}} \leqslant 10^{6}$ the boundary-layer mode is more unstable than the mushy-layer mode. For $T_{\mathrm{a}}=5 \times 10^{7}$, the boundary-layer mode is more stable when $\mathscr{A}<0.28$ and becomes more unstable otherwise. Similar result is obtained for $T_{\mathrm{a}} \times 10^{8}$, the switch of the critical mode occurs at $\mathscr{A}=0.51$.

To examine the rotation effect on the stability of the two convection modes, we show in Fig. 8 the $R_{\mathrm{m}}^{\mathrm{c}}$ of both the boundary-layer and the mushy-layer modes of four different $\mathscr{A}(0,0.1,0.3,0.5)$. For $\mathscr{A}=0$ [Fig. 8(a)], the $R_{\mathrm{m}}^{\mathrm{c}}$ of the mushy-layer mode does not exist for $T_{\mathrm{a}} \geqslant 10^{4}$ so that only the $R_{\mathrm{m}}^{\mathrm{c}}$ of the boundarylayer mode is shown in the figure, which is monotonically increasing with $T_{\mathrm{a}}$. For $\mathscr{A}=0.1$ [Fig. 8(b)], the $R_{\mathrm{II}}^{\mathrm{c}}$ of the mushy-layer mode does not exist until $T_{\mathrm{a}} \geqslant 10^{6}$; the convection mode becomes the critical one at $T_{\mathrm{a}} \approx 2 \times 10^{7}$. The $R_{\mathrm{m}}^{\mathrm{c}}$ for both modes increase monotonically with $T_{\mathrm{a}}$. For $\mathscr{A}=0.3$ [Fig. 8(c)] and 0.5 [Fig. 8(d)], the $R_{\mathrm{m}}^{\mathrm{c}}$ of the boundary-layer mode increases monotonically with $T_{\mathrm{a}}$ while that of the mushy-layer mode decreases slightly with $T_{\mathrm{a}}$ first, and turns into an increase function of $T_{\mathrm{a}}$ at $T_{\mathrm{a}} \approx 10^{6}$. For these two cases of larger $\mathscr{A}$, the mushy-layer mode 
(a)

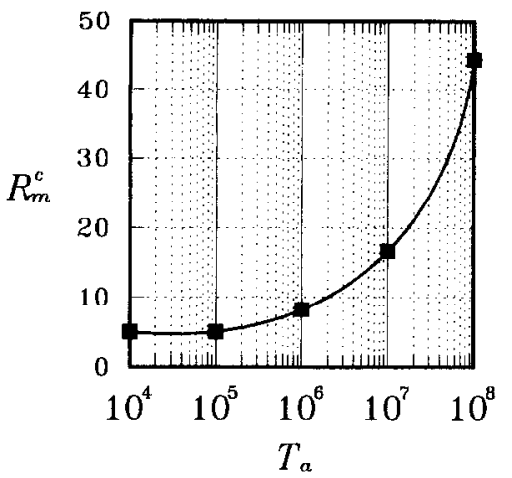

(c)

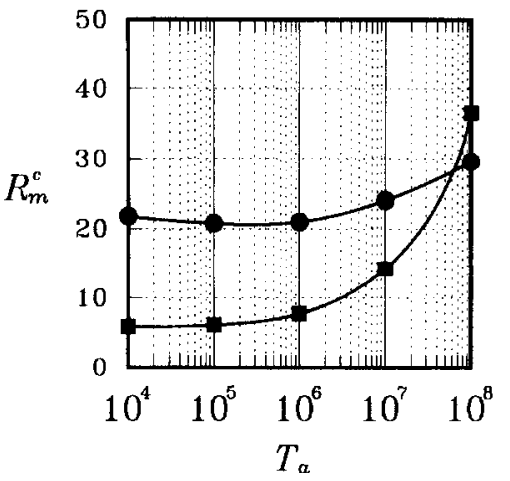

(b)

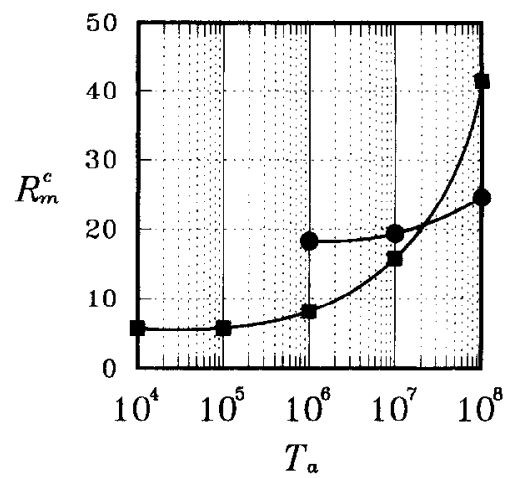

(d)

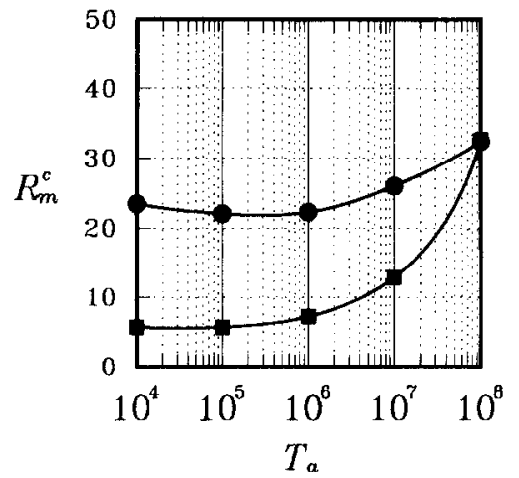

Fig. 8. The variations of $R_{\mathrm{m}}^{\mathrm{c}}$ with $T_{\mathrm{a}}$ for four different $\mathscr{A}:$ (a) $\mathscr{A}=0 ;$ (b) $\mathscr{A}=0.1$; (c) $\mathscr{A}=0.3$; (d) $\mathscr{A}=0.5 .-$, the mushy-layer mode; -

-, the boundary-layer mode.

replaces the boundary-layer mode, becoming the most critical one as $T_{\mathrm{a}}$ increases up to $3.4 \times 10^{7}$ and $9.9 \times 10^{7}$, respectively.

Based on the results shown above, the effects of rotation on the convection modes can be summarized as follows. First, to the mushy-layer mode, rotation does not influence significantly the $\alpha^{\mathrm{c}}$ for all $\alpha$ considered. For $\mathscr{A} \leqslant 0.1$ rotation enhances the stability (or increases the $R_{\mathrm{m}}^{\mathrm{c}}$ ) but slightly. For $\mathscr{A} \geqslant 0.3$ rotation leads to a smaller $R_{\mathrm{m}}^{\mathrm{c}}$ when $T_{\mathrm{a}}<10^{6}$, but results in a larger $R_{\mathrm{m}}^{\mathrm{c}}$ otherwise. Secondly, to the boundary-layer mode, rotation induces a slight increase for both $x^{c}$ and $R_{\mathrm{m}}^{\mathrm{c}}$ when $\mathscr{A}=0$. For $\mathscr{A} \geqslant 0.1$, both $\alpha^{\mathrm{c}}$ and $R_{\mathrm{m}}^{\mathrm{c}}$ increase largely with $T_{\mathrm{a}}$, especially at $T_{\mathrm{a}} \geqslant 10^{6}$. Thirdly, for $\mathscr{A}=0$, rotation stabilizes the mushy-layer mode more than the boundary-layer mode; which is opposite for $\mathscr{A}>0$. Finally, and maybe the most important remark, rotation may switch the convection mode from one to the other. To illustrate this remark, we summarize the $T_{a}^{*}$, the critical $T_{\mathrm{a}}$ at which the change of convection mode occurs, for various $\mathscr{A}$ in Fig. 9. For $\mathscr{A}=0$, the critical mode changes from mushy-layer mode into boundary-layer mode at $T_{\mathrm{a}} \approx 11$. For $\mathscr{A}>0$, the boundary-layer mode prevails when $T_{\mathrm{a}}<10^{7}$. The value of $T_{\mathrm{a}}^{*}$ generally lies between $7 \times 10^{6}$ and $1 \times 10^{8}$ when $0<\mathscr{A} \leqslant 0.5$. Beyond $T_{\mathrm{a}}^{*}$ the critical mode is the mushy-layer mode except $\mathscr{A}=0$.

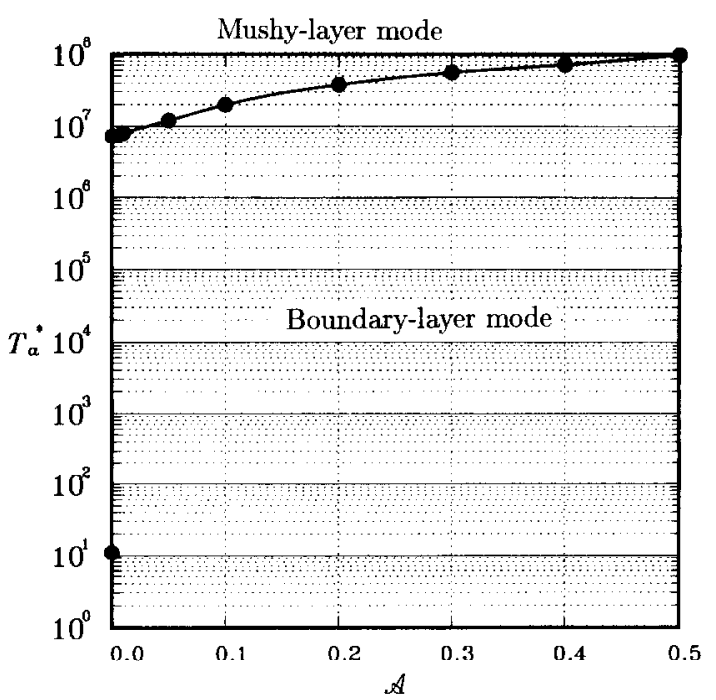

Fig. 9. The critical $T_{\mathrm{a}}^{*}$ at which the switch of the convection mode occurs. For $\mathscr{A}>0$, the region under the curve is for the boundary layer mode and the region above the curve is for the mushy-layer mode. For $\mathscr{A}=0$, the mushy-layer mode is the most unstable when $T_{\mathrm{a}}<11$, shown by a solid circle, and the boundary-layer mode is the most unstable otherwise.

The above remarks reflect the fact that the rotation effects on the two convection modes are quite different, depending largely on the $\mathscr{A}$ of the alloy 
considered. The explanation is provided in the following. To the mushy-layer mode, the convection occurs mostly in the mushy region in which the solutal diffusivity is so small that the cases of $\mathscr{A}=0$ and $\mathscr{A} \neq 0$ are essentially the same since the double-diffusive effect does not exist in the mush. This can be seen from the results shown in Figs. 8(b)-(d), if only the $R_{\mathrm{m}}^{\mathrm{c}}$ of the mushy-layer mode exists, their values are very close for all $\mathscr{A}$ considered ; so are the $\alpha^{\mathrm{c}}$ of different $\mathscr{A}$. If the difference must be precisely pointed out, one can find that for a larger $T_{\mathrm{a}}$, a larger $R_{\mathrm{m}}^{\mathrm{c}}$ is obtained; which obviously is due to the great stabilizing effect of the rotation on the boundary-layer mode when $T_{\mathrm{a}}$ is large.

To the boundary-layer mode, on the other hand, rotation stabilizes the motionless basic state much largely for $\mathscr{A}>0$ than $\mathscr{A}=0$. The explanation is more complex. For a compositional convection case $\mathscr{A}=0$, the buoyant flow containing less concentrated fluid moves upwards above the melt-mush interface. The motion is retarded mostly by the viscous force and slightly by the solutal diffusion. Accordingly, the thickness of the compositional boundary-layer becomes large and so does the critical wavelength. Moreover, rotation induces an Ekman layer above the melt-mush interface [17]. The horizontal flow in the Ekman layer acts as a centripetal force, suppressing the motion of the boundary-layer mode and leading to a more stable state. For $\mathscr{A}=0$, however, the thickness of Ekman layer is relatively smaller than the compositional boundary-layer, the suppression turns out to be small.

For the double-diffusive case $\mathscr{A}>0$, the buoyant flow is retarded not only by the viscous force and the solutal diffusion, but also, and in fact largely, by the thermal gradient, which is the stabilizing mechanism to the present double-diffusive system. Consequently, the thickness of the compositional boundary-layer (or the critical wavelength) is smaller than the case of $\mathscr{A}=0$ and is also influenced more intensively by rotation because the thickness of Ekman layer now is about the same with the characteristic length of the boundary-layer mode. As shown in Fig. 8, the influence of the Ekman layer becomes more significant when rotation is more intensive.

Both the investigations of Kuo et al. [15] and Neilson and Incropera [17] showed that the rotation speed up to $120 \mathrm{rpm}$ is not sufficient to prohibit the formation of freckles. Results shown in Fig. 8 may shed light on the reason. For alloys of different $\mathscr{A}$, rotation does not increase the value of $R_{\mathrm{m}}^{\mathrm{c}}$ significantly until $T_{\mathrm{u}}>10^{6}$, which is equivalent to $1.24 \times 10^{5} \mathrm{rpm}$ for a $\mathrm{NH}_{4} \mathrm{Cl}$ solution [22]. Accordingly, one may expect that rotation will not effectively prohibit the formation of freckles unless the angular velocity is extremely high. Nevertheless, a rotation about an axis with an inclined angle [3] or a intermittent rotation with a spin-up and spin-down cycle [17] can effectively prohibit the formation of freckles, both merit further study in the future.

\section{CONCLUDING REMARKS}

From above discussion on the results obtained from the linear stability analysis, focusing especially on the competition of the boundary-layer and mushy-layer modes, several concluding remarks can be drawn as follows.

(1) Uniform rotation about a vertical axis may not be an effective method to prohibit the formation of freckles unless the rotation speed is sufficiently high.

(2) Rotation may induce the change of convection mode, and in turn influences the stability characteristics of the subsequent plume convection or the formation of freckles.

(3) The effects of rotation on the stability of convection modes depend largely on the value of $\mathscr{A}$, a physical parameter influencing the intrinsic characteristics of the convection.

(4) For an alloy of $\mathscr{A}>0$ (suitable for most of the alloys in nature), rotation stabilizes more the boundary-layer mode than the mushy-layer mode. As $T_{\mathrm{a}}>7 \times 10^{6}$, the mushy-layer mode becomes the most critical one. Under such a circumstance, the plume convection (a result of the mushy-layer mode convection) may occur without a salt-finger (boundarylayer mode) convection beforehand.

Acknowledgement - -The financial support for this work from Nation Science Council through Grant No. NSC 85-2212E-002-010 are gratefully acknowledged.

\section{REFERENCES}

I. S. M. Copley, A. F. Giamei, S. M. Johnson and M. F. Hornbecker, The origin of freckles in undirectionally solidified castings, Metall. Trans. 1, 2193 (1970).

2. S. R. Coriell, M. R. Cordes, W. J. Boettinger and R. F. Sekerka, Convective and interfacial instability during unidirectional solidification of a binary alloy, $J$. Crystal Growth 49, 13 (1980).

3. A. K. Sample and A. Hellawell, The mechanisms of formation and prevention of channel segregation during alloy solidification, Metall. Trans. A, 15, 2163 (1984)

4. D. G. Neilson and F. P. Incropera, Numerical study of the effects on transport phenomena on macrosegregation during directional solidification of a binary substrate, Wärme- und Stoffübertragung (Thermo. Fluid Dyn.) 27(1), 1 (1991).

5. S. D. Felicelli, J. C. Heinrich and D. R. Poirier, Simulation of freckles during vertical solidification of binary alloys, Metall. Trans B 22, 847 (1991).

6. C. F. Chen and F. Chen, Experimental study of directional solidification of aqueous ammonium chloride solution, J. Fluid Mech. 227, 567 (1991).

7. S. Tait and C. Jaupart, Compositional convection in a reactive crystalline mush and melt differentiation, $J$. Geophys. Res. 97, 6735 (1992).

8. M. G. Worster, Instabilities of the liquid and mushy regions during solidification of alloys, J. Fluid Mech. 237, 649 (1992).

9. A. Hellawell, J. R. Sarazin and R. S. Steube, Channel convection in partly solidified systems, Phil. Trans. Soc. Lond. A 345, 507 (1993).

10. G. Amberg and G. M. Homsy, Nonlinear analysis of 
buoyant convection in binary solidification with application to channel formation, $J$. Fluid Mech. 252, 79 (1993).

11. P. W. Emms and A. C. Fowler, Compositional convection in the solidification of binary alloys, $J$. Fluid Mech. 262, 111 (1994).

12. M. G. Worster and R. C. Kerr, The transient behaviour of alloys solidified from below prior to the formation of chimneys, J. Fluid Mech. 269, 23 (1994).

13. F. Chen, J. W. Lu and T. L. Yang, Convective instability in directional solidification of ammonium chloride solutions cooling from below, J. Fluid Mech. 276, 163 (1994).

14. E. O. Schulz-DuBois, Accelerated crucible rotation: hydrodynamics and stirring effect, J. Crystal Growth 12, 81 (1972).

15. S. Kuo, D. R. Poirier and M. C. Flemings, Macrosegregation in rotated remelted ingots, Metall. Trans. $B, 9,2163$ (1978).

16. A. Oztekin and A. J. Pearlstein, Coriolis effects on the stability of plane-front solidification of dilute $\mathrm{Pb}-\mathrm{Sn}$ binary alloys, Metall. Trans B 23, 73 (1992).

17. D. G. Neilson and F. P. Incropera, Effect of rotation on fluid motion and channel formation during unidirectional solidification of a binary alloy, Int. J. Heat Mass Transfer 36(2), 489 (1993).

18. G. S. Beavers and D. D. Joseph, Boundary conditions at a naturally permeable wall, J. Fluid Mech. 30, 197 (1967).

19. S. Chandrasekhar, Hydrodynamic and Hydromagnetic Stability. Clarendon Press, Oxford (1961).

20. W. Weber, G. Neumann and G. Muller, Stabilizing influence on the Coriolis force during melt growth on a centrifuge, J. Crystal Growth 100, 145 (1990).

21. J. S. Turner, Buoyancy Effects in Fluids. Cambridge University Press, Cambridge (1979).

22. F. Chen, T. L. Yang and J. W. Lu, Influence of convection on solidification of binary solutions cooling from below, J. Appl. Phys. 74(12), 7531 (1993). 